\title{
High-Q optical resonators in silicon-on-insulator-based slot waveguides
}

\author{
Tom Baehr-Jones, Michael Hochberg, Chris Walker, and Axel Scherer ${ }^{a)}$ \\ California Institute of Technology, Pasadena, California 91125
}

(Received 1 July 2004; accepted 6 January 2005; published online 14 February 2005)

\begin{abstract}
This letter describes the design, fabrication and characterization of high- $Q$ oval resonators based on slot waveguide geometries in thin silicon-on-insulator material. Optical quality factors of up to 27000 were measured in such filters, and we estimate losses of $-10 \mathrm{~dB} / \mathrm{cm}$ in the slotted waveguides on the basis of our resonator measurements. Such waveguides enable the concentration of light to very high optical fields within nanoscale dimensions, and show promise for the confinement of light in low-index material with potential applications for optical modulation, nonlinear optics and optical sensing. (C) 2005 American Institute of Physics.
\end{abstract}

[DOI: $10.1063 / 1.1871360]$

It has been known for some time that low loss waveguides can be fabricated in thin silicon-on-insulator (SOI) structures. ${ }^{1}$ One can then form high quality factor ring or oval resonators in these structures. ${ }^{2-4}$ In these SOI waveguides, vertical confinement of light is obtained from the index contrast between the silicon core and the low index cladding and the buried silicon dioxide layer, whereas lateral confinement can be obtained by lithographically patterning the silicon. The majority of the light tends to be guided within the silicon core in such waveguide. Although the high refractive index contrast between silicon and its oxide provide excellent optical confinement, guiding within the silicon core can be problematic for some applications. In particular, at very high optical intensities, two-photon absorption in the silicon may lead to high optical losses. Moreover, it is often desirable to maximize the field intensity overlap between the optical waveguide mode and a lower index cladding material, especially when that cladding is optically active and provides electro-optic modulation ${ }^{5}$ or chemical sensing.

A possible solution to these problems consists of using a slot waveguide geometry, recently described by Almeida et $a l .{ }^{6}$ The essential feature of such a slot waveguide is that two silicon stripes are formed by etching an SOI slab, and are separated by a small distance of approximately $60 \mathrm{~nm}$. The optical mode in such a structure tends to propagate mainly within the center of that waveguide. In the case of primarily horizontal polarization, the discontinuity condition at the cladding-silicon interface leads to a large concentration of the optical field in the trench; we predict that the electric field intensity would be roughly $10^{8} \sqrt{P} \mathrm{~V} / \mathrm{m}$ where $P$ is the input power in watts. Figure 1 shows the approximate geometry used for the design in this letter, as well as the solved mode pattern for light at approximately $1.53 \mu \mathrm{m}$. This mode was obtained from a full vectoral eigensolver based on a finite difference time domain model. ${ }^{7}$ Our designs use a 120 $\mathrm{nm}$ silicon-on-insulator layer and 300-nm-wide silicon strips on top of a 1.4- $\mu \mathrm{m}$-thick buried oxide layer, which is in turn deposited on a silicon substrate. After manually cleaving the wafers, the pieces were spin cleaned with acetone and isopropanol and then air dried. Hydrogen silsesquioxane (HSQ) resist from Dow Corning Corporation, at 2\% concentration,

\footnotetext{
a) Author to whom correspondence should be addressed; electronic mail:
} etcher@caltech.edu was spun on at $1000 \mathrm{rpm}$ and baked for $4 \mathrm{~min}$ at $180{ }^{\circ} \mathrm{C}$. Devices were exposed in a Leica EBPG 5000+ electron beamwriter at $100 \mathrm{kV}$ at a dose of $3500 \mu \mathrm{C} / \mathrm{cm}_{2}$ and developed in MIF-300 developer for 3 min. Etching was performed with an Oxford Plasmalab 100 inductively coupled plasma (ICP) reactive ion etching machine with 12 mTorr of chlorine, $800 \mathrm{~W}$ of ICP power, and $50 \mathrm{~W}$ of forward power all applied for $33 \mathrm{~s}$. After the lithographic waveguide definition process, poly(methylmethacrylate) was deposited as the top cladding layer. We experimented with various widths for the central slot, testing devices with 50, 60 and $70 \mathrm{~nm}$ gaps. The mode profile and dispersion diagram shown in Fig. 1 are for a $60 \mathrm{~nm}$ slot.

In the 1.4-1.6 $\mu \mathrm{m}$ wavelength regime, it should be noted that this waveguide geometry is single mode, and a wellcontained optical mode is supported between the two silicon waveguide slabs. There is some loss that such an optical mode will experience even in the absence of any scattering loss or material absorption due to leakage of light into the silicon substrate. This substrate loss can be estimated semianalytically via perturbation, ${ }^{8}$ and ranges from approximately $-0.15 \mathrm{~dB} / \mathrm{cm}$ at $1.49 \mu \mathrm{m}$ to about $-0.6 \mathrm{~dB} / \mathrm{cm}$ at $1.55 \mu \mathrm{m}$ in this spectral range for our particular SOI wafer geometry.

Oval resonators were fabricated by patterning the slot waveguides into an oval shape. We chose an oval resonator geometry over the more conventional circular shape to enable a longer coupling distance between the oval and the external coupling waveguide. Slots were introduced into both the oval and external coupling waveguides, and Fig. 2 shows scanning electron micrograph images of the resonators and their input couplers.

Many different coupling lengths and ring to input waveguide separations were fabricated and tested, as it was difficult to predict the exact coupling strength for any given geometry. Moreover, the waveguide losses of the slot waveguides could not be predicted before fabricating the devices, and it is well known that the most distinct resonance behavior would be observed for critically coupled resonators, in which the coupling strength roughly matches the round trip loss in the ring.

It is useful to present an analytic expression for the quality factor of a ring resonator ${ }^{2}$ 


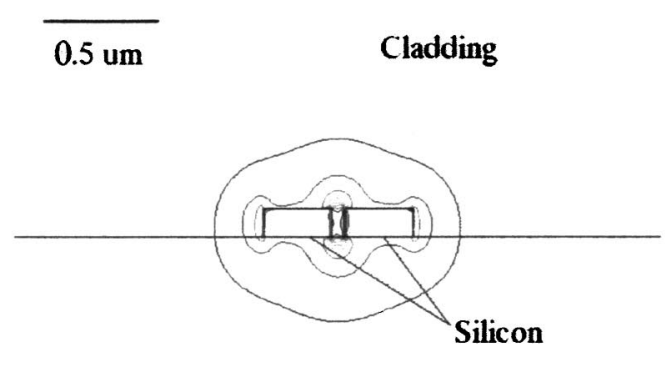

Buried Oxide

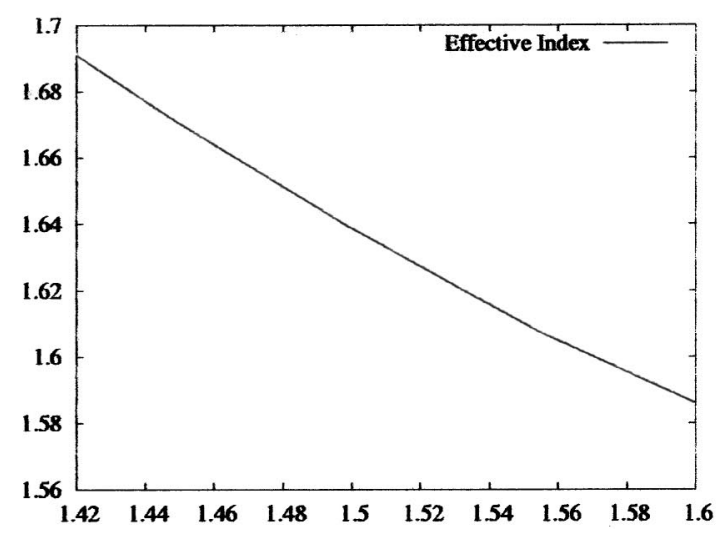

FIG. 1. Slot waveguide mode profile, and effective index vs free space wavelength in microns. The mode profile consists of $|E|$ contours, plotted in increments of $10 \%$ of the max field value. The $E$ field is oriented primarily parallel to the wafer surface.

$$
Q=\frac{\pi L}{\lambda_{0}} \frac{\left(n_{0}-\lambda_{0}\left(\frac{\partial n}{\partial \lambda}\right)_{0}\right)}{(1-\alpha t)} .
$$

Also, the free spectral range can be calculated via

$$
\Delta \lambda=\frac{\left(\frac{\lambda_{0}}{L}\right)}{\left(\frac{1}{L}+\frac{n_{0}}{\lambda_{0}}-\left(\frac{\partial n}{\partial \lambda}\right)_{0}\right)} .
$$

Here, $L$ is the round trip length in the ring, and $n_{0}$ and $\lambda_{0}$ are the index of refraction, and the wavelength at resonance, respectively. We take $\alpha$ to be the optical amplitude attenuation factor due to loss in a single trip around the ring, and $t$ to be the optical amplitude attenuation factor due to traveling past the coupling region. $(\partial n / \partial \lambda)_{0}$ is the derivative of the effective index with respect to the wavelength at the resonance peak, and it can be shown that this term is roughly equal to $-0.6 \mu \mathrm{m}^{-1}$ from the $1.4-1.6 \mu \mathrm{m}$ spectral range for the slot waveguides studied here.

We have observed a quality factor of 27000 in a device fabricated with a slot size of $70 \mathrm{~nm}$, a ring to input waveguide edge to edge separation of $650 \mathrm{~nm}$, and a coupling distance of $1.6 \mu \mathrm{m}$. The radius of the circular part of the slotted oval was $50 \mu \mathrm{m}$. This resonance was observed near $1488 \mathrm{~nm}$, and the resonance peak had an extinction ratio of $4.5 \mathrm{~dB}$. Figure 3 shows the measured transmission spectrum past the ring, normalized for the input coupler base line efficiency of our test system. Because the extinction ratio at the resonance peak was not very large in this case, it was not possible to accurately determine waveguide losses from this device. By measuring many devices with different geom- etries, we obtained data on resonators with higher extinction ratios that approached critical coupling. One such device was a $50 \mu \mathrm{m}$ radius slotted ring resonator with a $60 \mathrm{~nm}$ waveguide gap, a ring to input waveguide spacing of $550 \mathrm{~nm}$ and coupling length of $1.6 \mu \mathrm{m}$. In this device, a $Q$ of 23400 was observed near $1523 \mathrm{~nm}$, with an on-resonance extinction of $14.7 \mathrm{~dB}$.

Since this resonance is nearly critically coupled, the waveguide loss can be estimated using Eq. (1) as $-10 \mathrm{~dB} /$ $\mathrm{cm}$. We can also use Eq. (2) to further validate our theoretical picture of the ring resonator; the observed free spectral range of this resonator was $2.74 \mathrm{~nm}$, while Eq. (2) predicts $2.9 \mathrm{~nm}$. This discrepancy is most likely due to small differences in the fabricated dimensions as compared to those for which the numerical solutions were obtained. The fabricated devices were found to be within $10 \mathrm{~nm}$ of the drawn widths through metrology in a scanning electron microscope, and the sidewalls were found to be vertical. Nonetheless, because small changes in the width will change the optical path length around the ring, it is reasonable to attribute this discrepancy to small inconsistencies in the width of the guides.

To further validate the waveguide loss result, several waveguide loss calibration loops were fabricated with varying lengths of the slot waveguide, ranging from 200 to $8200 \mu \mathrm{m}$ in length. A total of five devices were studied for 50, 60 and $70 \mathrm{~nm}$ center slot waveguides each. Performing a linear regression on the peak transmission of each series yielded waveguide loss figures of $11.6 \pm 3.5 \mathrm{~dB} / \mathrm{cm}$ for the $50 \mathrm{~nm}$ center waveguide, $7.7 \pm 2.3 \mathrm{~dB} / \mathrm{cm}$ for the $60 \mathrm{~nm}$ center waveguide, and $8.1 \pm 1.1 \mathrm{~dB} / \mathrm{cm}$ for the $70 \mathrm{~nm}$ center waveguide. These figures are in agreement with the loss estimated from the oval resonator. Since the theoretical loss due to substrate leakage is much lower than this, it is clear
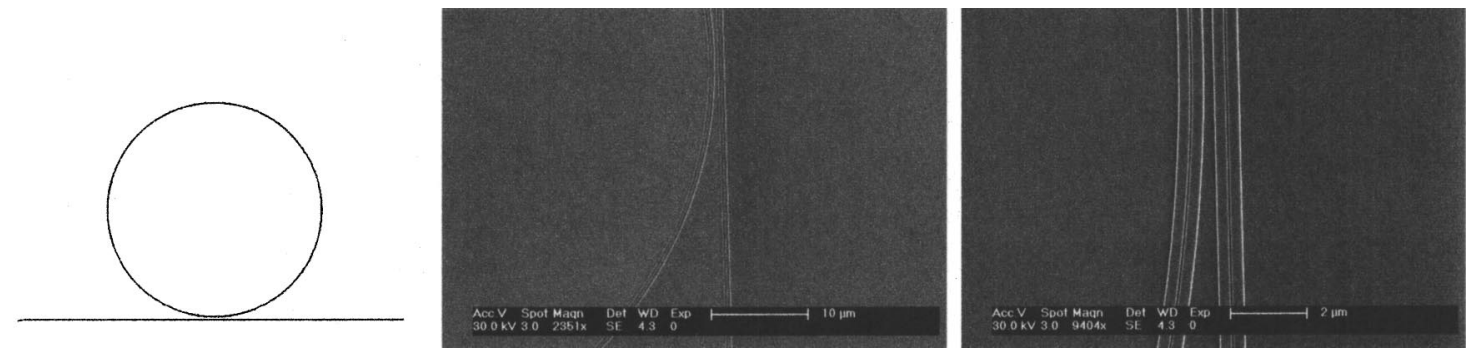

FIG. 2. Device layout, and scanning electron micrographs of the slot waveguide oval and input waveguide. The entire oval is shown in the middle frame, while a detailed image of the coupling region is shown in the right frame.

Downloaded 14 Dec 2005 to 131.215.225.171. Redistribution subject to AIP license or copyright, see http://apl.aip.org/apl/copyright.jsp 

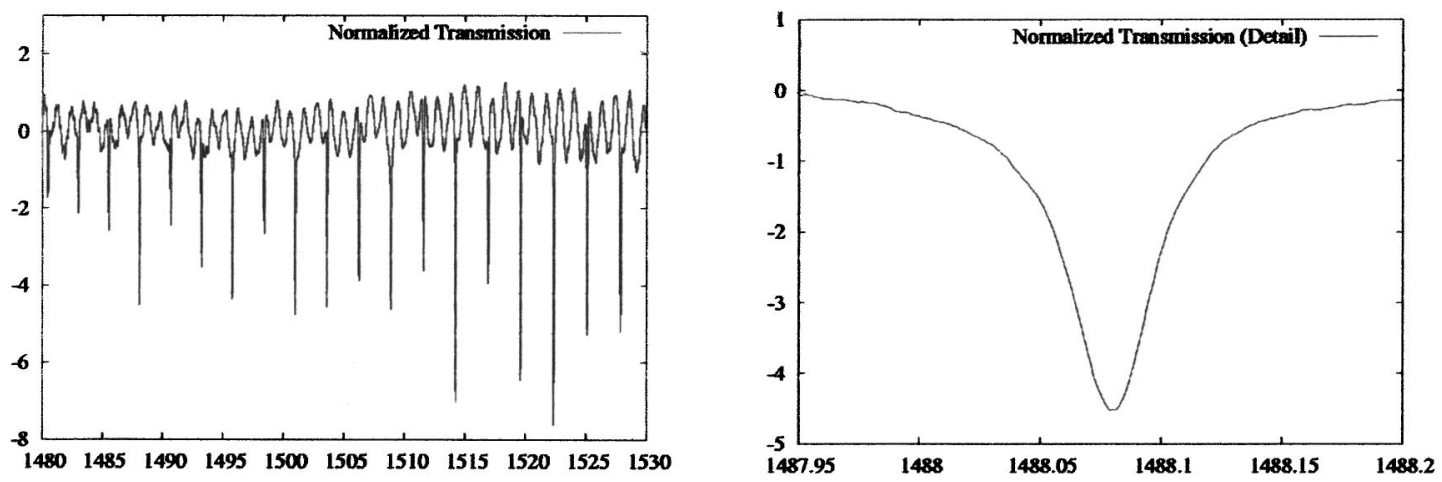

FIG. 3. Measured transmission spectrum in $\mathrm{dB}$ vs laser wavelength in $\mathrm{nm}$ past a high-quality factor slot ring resonator. The detail of the peak near $1488 \mathrm{~nm}$ is also shown.

that a great deal of loss is due to edge roughness and possibly material absorption. Edge roughness was characterized with electron microscopy and found to be less than $10 \mathrm{~nm}$ peak to valley. There is certainly the possibility for engineering improvements to decrease this loss further.

We have demonstrated high-quality factor resonators with a slotted waveguide geometry on thin silicon-oninsulator material. The high-quality factors observed suggest that slotted waveguides can have low loss, and ring resonators constructed from this waveguide geometry will find applications as chemical and biological sensors and tunable telecommunications filters. In particular, for sensing and modulation applications as well as use in nonlinear optics, the high optical field concentration that can be supported in the cladding material of the slotted waveguide geometry will be very advantageous when compared to more conventional index guides.
The authors would like to acknowledge generous support from DARPA and NAVAIR under contract No. N0042102-D-3223 and from the AFOSR.

${ }^{1}$ T. Baehr-Jones, M. Hochberg, C. Walker, and A. Scherer, Appl. Phys. Lett. (submitted)

${ }^{2}$ D. Armani, T. Kippenberg, S. Spillane, and K. Vahala, Nature (London) 421, 925 (2003).

${ }^{3}$ B. E. Little, S. T. Chu, W. Pan, and Y. Kokubun, IEEE Photonics Technol. Lett. 12, 323 (2000).

${ }^{4}$ D. Rafizadeh, J. P. Zhang, R. C. Tiberio, and S. T. Ho, J. Lightwave Technol. 16, 1308 (1998).

${ }^{5}$ L. R. Dalton, B. H. Robinson, A. K. Y. Jen, W. H. Steier, and R. Nielsen, Opt. Mater. (Amsterdam, Neth.) 21, 19 (2003).

${ }^{6}$ V. R. Almeida, Q. F. Xu, C. A. Barrios, and M. Lipson, Opt. Lett. 29, 1209 (2004).

${ }^{7}$ J. D. Joannopoulos, R. D. Meade, and J. N. Winn, Photonic Crystals (Princeton University Press, Princeton, 1995).

${ }^{8}$ T. Baehr-Jones, M. Hochberg, and A. Scherer, J. Lightwave Technol. (submitted). 